\title{
Acknowledgement to Reviewers of Cancers in 2017
}

\section{Cancers Editorial Office}

MDPI AG, St. Alban-Anlage 66, 4052 Basel, Switzerland; cancers@mdpi.com

Published: 10 January 2018

Peer review is an essential part in the publication process, ensuring that Cancers maintains high quality standards for its published papers. In 2017, a total of 172 papers were published in the journal. Thanks to the cooperation of our reviewers, the median time to first decision was 21 days and the median time to publication was 44 days. The editors would like to express their sincere gratitude to the following reviewers for their time and dedication in 2017:

Advani, Sunil

Aggarwal, Bharat B.

Albano, Emanuele

Alevizopoulos, Konstantinos

Alkan, Serhan

Allen, Christopher

Amato, Rosario

Amato, Robert J.

Amrutkar, Manoj

An, Sai

Andersen, Jens Rikardt

Ansa, Benjamin

Antonescu, Costin

Arfuso, Frank

Argani, Pedram

Arora, Vivek K.

Asiedu, Michael

Atherly, Adam

Aveic, Sanja

Avila, Matias A.

Azmi, Asfar S.

Babib, Amyn

Bagnasco, Annamaria

Bai, Shuhua

Baniahmad, Aria

Bansal, Ajay

Barbagallo, Davide

Barberi-Heyob, Muriel A.

Barbolina, Maria V.

Basu, Alakananda

Battistelli, Cecilia

Battistini, Lucia

Bayascas, Jose

Beaman, Kenneth D.

Becker, Therese M.

Beebe, Stephen J.
Beierle, Elizabeth

Beltran, Chris

Benistant, Christine

Benjamin, Don

Bentel, Jacqueline M.

Bergamo, Alberta

Bergh, Anders

Bergmann, Andreas

Bigey, Pascal

Binder, Mascha

Bizzarri, Mariano

Black, Esther P.

Blons, Hélène

Boddicker, Rebecca L.

Bodnar, Lubomir

Boehme, Karl

Bonzheim, Irina

Booken, Nina

Bora-Singhal, Namrata

Bordonaro, Michael

Borrebaeck, Carl A.K.

Boström, Jan Patrick

Bouffler, Simon

Bourgeois-Daigneault, Marie-Claude

Bournet, Barbara

Bown, Stephen

Brandao, Joao

Breckpot, Karine

Breitenbücher, Frank

Buchholz, Julia

Burman, Pia

Calin, George

Calvisi, Diego F.

Cao, Bo

Carosella, Edgardo D.

Carpinteiro, Alexander 
Carter, David

Carver, Brett $\mathrm{S}$.

Cerchia, Laura

Ceresa, Brian

Chalmers, Jeffrey J.

Chandrasiri, Upeksha

Chantrill, Lorraine A.

Chatterjee, Jhinuk

Chen, Zhuo (Georgia)

Chen, Kun-Ming

Chen, Zhong

Chevret, Edith

Choi, Yoon-La

Chou, Cheng-Yang

Choudhury, Malay

Choulier, Laurence

Christensen, James G.

Chung, Eun Joo

Cicenas, Jonas

Clark, Geoffrey

Clere, Nicolas

Coley, Helen M.

Coller, Hilary

Colson, Natalie

Cooke, Marcus

Coppedè, Fabio

Corn, Paul

Cortese, Katia

Crnogorac-Jurcevic, Tatjana

Curtis, Sarah

Dal Corso, Alberto

Dalby, Kevin N.

Damia, Giovanna

Davies, Michaell

Davis, Richard

De, Pradip

Degrassi, Francesca

Devys, Didier

DeWees, Todd

Dey, Nandini

Dharmarajan, Arun

Di Blasio, Laura

Di Fazio, Pietro

Dormond, Olivier

Dovat, Sinisa

Durham, Andrew L.

Duvvuri, Umamaheswar

Dy, Grace

Eid, Nabil

El-Heliebi, Amin

Engelberg, David

Faber, Anthony C.
Feng, Hui

Feng, Liu-Smith

Ferretti, Elisabetta

Filleur, Stephanie

Fitzgerald, Rebecca C.

Foijer, Floris

Font-Burgada, Joan

Franchina, Tindara

Freeman, James W

Fribley, Andrew M.

Frochot, Céline

Fujita, Mitsugu

Funel, Niccola

Furukawa, Tatsuhiko

Futakuchi, Mitsuru

Gao, Yang

Gao, Shuai

García-Jiménez, Custodia

Gatault, Solène

$\mathrm{Ge}$, Xiaodong

Georgakilas, Alexandros

Ghigo, Alessandra

Giardino, Alessandro

Giuriato, Sylvie

Gius, David

Gobe, Glenda C.

Godar, Dianne E.

Gohda, Eiichi

Golovleva, Irina

Gonzalez, Victor

González-González, Rogelio

Grant, William

Gray, Steven

Grivas, Petros

Gross, Gideon

Grusch, Michael

Guedan, Sonia

Guha, Prajna P.

Guillermet-Guibert, Julie

Gullberg, Donald

Guo, Zong Sheng

Gustafsson, Jan-Åke

Hamanishi, Junzo

Hamieh, Mohamad

Hamilton, Gerhard

Hannafon, Bethany

Haque, Inamul

Harbin, Sherry L.

Hariharan, Seethalakshmi

Hassan, Mohamed

Hata, Yutaka

Hatzakis, Nikos 
Heckman, Caroline

Hellerbrand, Claus

Heng, Henry

Hildreth, Blake

Hiraoka, Nobuyoshi

Ho, Yeung

Hofman, Paul

Hojman, Pernille

Holinstat, Michael

Holstein, Sarah

Hori, Toshiyuki

Hsieh, James J.

$\mathrm{Hu}$, Dong Gui

Hung, Jan-jong

Huppi, Konrad

Husain, Siraj

Hutchison, Robert E.

Ignatius, Sai-Hong

Ilmer, Matthias

Inamura, Kentaro

Inga, Alberto

Iorio, Marilena

Grand, Roger J.

Jacobs, Aaron T.

Jacola, Lisa

James, McCaul

Jang, Se Jin

Jankovic, Momcilo

Jardin, Isaac

Jasiulionis, Miriam G.

Jean, Didier

Jeggo, Penny

Jenkins, Samir V.

Jensen, Randy L.

Jensen, Lars Henrik

Jeong, Keun-Yeong

Jia, William

Jiang, Chen Chen

Jiang, Jianxiong

Johansson, Staffan

Jung, Seungyoun

Jung, Klaus

Lauf, Peter K.

Kallis, Yiannis

Kallunki, Tuula

Kanai, Masashi

Kang, Hyunseok

Kashiwagi, Shinichiro

Kasinski, Andrea

Katiyar, Santosh K.

Kawasumi, Masaoki

Kelly, Janet
Khan, Shafiq

Kibriya, Muhammed

Kim, Chang Hyeuk

Kim, Sang-We

Kim, Joongsun

Kim, Tae Il

Kim, Sang-We

Kim, Junghoon

Kim, Hyungjin

Klampfer, Lidija

Klapdor, Rüdiger

Kleeff, Jörg

Klokov, Dmitry Y.

Kobayashi, Junya

Kohno, Takashi

Konishi, Hirotaka

Kornprat, Peter

Kosaka, Nobuyoshi

Kosinsky, Robyn Laura

Koul, Hari

Kovacs, Pamela J.

Krishna, Somashekar G.

Krog Frandsen, Stine

Krug, Sebastian

Kubo, Toshio

Kurita, Hirofumi

Kurozumi, Kazuhiko

Kwong, Joseph

Kypta, Robert

La Vecchia, Carlo

Lachenmeier, Dirk

Lahooti, Hooshang

Lai, Hsueh-Chou

Lai, Raymond

Languino, Lucia $R$.

Lathia, Justin

Laura, Mansi

Laurenzana, Anna

Leavenworth, Jianmei

Lee, Kin Wah Terence

Lee, Jung-Min

Lee, Sang-Han

Leenstra, Sieger

Lele, Shashikant

Lengerke, Claudia

Leong, Hon

Leong-Poi, Howard

Li, Feng

Li, Calvin

Xiao, Lifu

Lin, Kui

Lin, Sue-Hwa 
Lin, Haibo

Lin, Huey-Jen

Lindqvist, Lisa

Liu, Botao

Liu, Jun-Jen

Liu, Delong

Liu, Yongqing

Liu, Yen-Nien

Locati, Laura D.

Luftig, Micah

Lumniczky, Katalin

Lundholm, Marie Lundholm

Lundstrom, Kenneth

Luo, Yong-Zhang

Luscinskas, Francis W.

Ma, Patrick

Mace, Thomas

Maffucci, Tania

Maggiolini, Marcello

Mahimainathan, Lenin

Månsson, Christopher

Maréchal, Alexandre

Marimpietri, Danilo

Martelli, Alberto

Martin, Sally K.

Martinon, Fabio

Masuelli, Laura

Matsugaki, Aira

Matsui, Junji

Maugeri, Marco

Mawhinney, Thomas

McClelland, Sarah

McKeague, Maureen

Mckillop, Iain H.

Medin, Jeffrey A.

Medová, Michaela

Mehta, Shwetal

Meijerink, Martijn R.

Mendenhall, Nancy

Menon, Smitha

Miller, Jeffrey S.

Miller, Alexandra C.

Miyoshi, Eiji

Mo, Yin-Yuan

Modjtahedi, Helmout

Morales, Julio

Morioka, Sho

Mudryj, Maria

Muñoz, Aránzazu Sánchez

Murray, Paul G.

Muto, Manabu

Nadiminty, Nagalakshmi
Nagaraju, Ganji Purnachandra

Nagel, Zachary

Nakamura, Asako

Nakaseko, Chiaki

Nandi, Saikat

Narita, Norihiko

Natarajan, Sathish Kumar

Nazarian, Javad

Neureiter, Daniel

Nevzorova, Yulia A.

$\mathrm{Ni}$, Xiao

Nishimura, Noriyuki

Nock, Nora

Nowsheen, Somaira

O'reilly, Eileen $\mathrm{M}$.

Ogino, Shuji

Oosting, Sjoukje F.

Ordónez-Mena, José M.

Oschlies, Ilske

Ove, Roger

Pagel, Marty

Paggetti, Jérôme

Palmer, Ruth $\mathrm{H}$.

Palmirotta, Raffaele

Parrington, John

Pastor, Fernando

Pedraza-Chaverri, José

Pellat-Deceunynck, Catherine

Peña, Maria Marjorette O.

Pinzani, Pamela

Plymate, Stephen R.

Pocheć, Ewa

Ponz-Sarvisé, Mariano

Porta, Camillo

Posey, Avery D.

Potiron, Vincent

Primo, Luca

Pulliero, Alessandra

Pyka, Thomas

Qian, Hong

Quintanilla-Martinez, Leticia

Rahib, Lola

Raimondo, Stefania

Rainero, Elena

Ramakrishnan, Sadeesh

Rassidakis, George Z.

Reales-Calderón, Jose Antonio

Redmer, Torben

Redondo Muñoz, Javier

Renaudineau, Yves

Repasky, Elizabeth A.

Ricci, Giulia 
Rich, Jeremy N.

Rizvi, Imran

Robles, Ana

Rödel, Franz

Rodriguez-Lafrasse, Claire

Romani, Andrea

Rooj, Arun

Rooman, Ilse

Roos, Wynand P.

Rosell, Rafael

Rosenzweig, Allison

Rübe, Claudia E.

Russell, Ryan

Sabatini, Linda M.

Saha, Achinto

Saini, Shikha

Saini, Sharanjot

Sainz Jr., Bruno

Salaspuro, Mikko

Saltman, David L.

Samuel, Temesgen

Sánchez-Tilló, Ester

Santoni-Rugiu, Eric

Sarkar, Saswata

Scaltriti, Maurizio

Schepisi, Giuseppe

Schildgen, Oliver

Scovassi, Anna Ivana

Di Sebastiano, Pierluigi

Seimiya, Hiroyuki

Shackelford, Rodney E.

Shafirstein, Gal

Shah, Nilay

Sharafeldin, Noha

Shen, Jia Zack

Shepherd, Peter

Sheth, Sandeep

Shiao, Stephen L.

Shim, Hyunsuk

Shinohara, Shogo

Shiozawa, Yusuke

Shirato, Hiroki

Shnyder, Steve

Sicard-Roselli, Cécile

Siegfried, Jill

Sihver, Lembit

Silva, Susana N.

Singh, Anil

Singh, Anurag

Singh, Shree Ram

Sklar, Larry A.

Skougaard, Kristin
Smahel, Michal

Smith, Robert A.

Soldevilla, Mario

Son, Deok-soo

Song, Jia L.

Sonis, Stephen

Sonnenberg, Arnoud

Sottnik, Joseph

Sprenger, Cynthia

Srivastava, Akhil

Srivenugopal, Kalkunte

Stangl, Herbert

Stefanini, Lucia

Steinbrunn, Torsten

Stewart, Teneale

Stickeler, Elmar

Stockmann, Christian

Stoker, Joshua B.

Stuber, Margaret

$\mathrm{Su}$, Rui

Sugii, Yuh

Sugimoto, Naotoshi

Sun, Qian

Sundar, Reshma

Sutcliffe, Julie L.

Suzuki, Keiji

Suzuki, Ryusuke

Swierczynski, Julian

Szegezdi, Eva

Taguchi, Yoshihiro

Taguchi, Ayumu

Takahashi, Akihisa

Takahashi, Hiroki

Takeuchi, Kengo

Takiguchi, Yuichi

Taniguchi, Hiroya

Tanner, Julian A.

Tarallo, Valeria

Tarasova, Nadya I.

Tartarone, Alfredo

Tashima, Toshihiko

Tatsuaki, Kanai

Testa, Ugo

Thomas, Sufi

Tinguely, Marianne

Tober, Kathleen

Torigoe, Toshihiko

Trembley, Janeen H.

Tremolada, Marta

Trøst Jørgensen, Jan

Tschan, Mario P.

Tsoulfas, George 
Turcotte, Lucie M.

Turner, Suzanne

Uhl, Eberhard

Van Den Bergh, Hubert

Van Den Brekel, Michiel Wilhelmus Maria

Van Etten, Jamie

VanderWeele, David

Vassetzky, Yegor

Vazquez, Marcelo

Vega, Francisco

Vella, Laura Jayne

Veltkamp, Christian

Venturella, Roberta

Verma, Navin Kumar

Viggiano, Davide

Villalobo, Antonio

Vincent, Vander Poorten

Viola, Giampietro

Vizeacoumar, Franco

Voutsadakis, Ioannis A.

Wahlestedt, Claes

Wakimoto, Hiroaki

Wang, Xiangdong

Ward, Douglas

Watson, Philip

Wedemeyer, Heiner

Weinberg, Robert A.

Weinhold, Niels

Westerhout, Ellen M.

Whelan, Rebecca

Whitelock, John

Willard, Victoria W.

William, William Nassib

Williams, Christopher C.

Winter, Jörn

Witkamp, Frederika Erica
Wu, Lily

$\mathrm{Wu}$, Xiaohua

Xie, Qi

Xing, Jianhua

Xing, Junji

Xiong, Zhaohui

Xiong, Zheng-Mei

$\mathrm{Xu}$, Baoshan

$\mathrm{Xu}$, Dazhong

Yaddanapudi, Kavitha

Yang, Pei

Yang, Sien-Sing

Yao, Jiayi

Yeh, Shiou-Hwei

Yip, Kay-pong

Yokoyama, Akira

Yu, Yan Ping

Yu, Yanlin

Yuan, Xue

Yuan, Chunyan

Yue, Junming

Zacksenhaus, Eldad

Zadeh, Gelareh

Zamay, Anna S.

Zaravinos, Apostolos

Zaret, Kenneth S.

Zhang, Caiguo

Zhang, Weijie

Zhang, Xiaodong

Zhou, Lan

Zhou, Wenchao

$\mathrm{Zi}$, Xiaolin

Ziegler, Patrick

Zigrino, Paola

$\mathrm{Zu}$, Youli

Zuco, Valentina

(C) 2018 by the authors. Licensee MDPI, Basel, Switzerland. This article is an open access article distributed under the terms and conditions of the Creative Commons Attribution (CC BY) license (http://creativecommons.org/licenses/by/4.0/). 